\title{
Kinetic PCR Analysis Using a CCD-camera and without Using Oligonucleotide Probes
}

\author{
Russell Higuchi and Robert Watson \\ Roche Molecular Systems \\ 1145 Atlantic Avenue, Alameda, Califronia 94501 \\ (510) $814-2901$
}

Higuchi, R. and Watson, R.M. (1999)

"Kinetic PCR analysis using a CCD-camera and without using oligonucleotide probes." in PCR methods manual (ed. M.A. Innis, D.H. Gelfand and JJ. Sninsky) pp 263-284

Academic Press, San Diego, CA 
Although this chapter will focus on the use of a CCD camera to monitor PCRs in the "dye-alone" mode, a brief overview is provided to explain the advantages and disadvantages of this approach compared to other methods of kinetic PCR analysis.

\section{Overview}

The real-time monitoring of PCR amplifications, or kinetic PCR analysis, allows one to follow PCR DNA replication on a cycle-by-cycle basis. This was first accomplished by including in the PCR at less than inhibitory levels a dye whose fluorescence increases upon binding dsDNA. Such a dye is the commonly used ethidium bromide (etbr) although many other dyes may be used. As an increasing amount of double-stranded DNA (dsDNA) is generated by the PCR, so is an increasing amount of fluorescence, which can be monitored from outside the reaction vessel. The initial use of this dye was in the qualitative detection of target sequences by the presence or absence of a fluorescence increase (Higuchi, et al. 1992). Subsequently, the cycle-by-cycle monitoring of fluorescence increase was shown to facilitate quantitation of the target sequence (Higuchi, et al., 1993). Compared with other methods of quantitation based on PCR, kinetic PCR has the advantages of being a "closed-tube" assay . These include mechanical simplicity, high thoughput and containment of potential carryover contamination. Kinetic PCR also has the ability to quanitify over a range of many logs of target concentration without the sample dilution needed by other methods.

Cycle-by-cycle monitoring allows one to trace a "growth curve" or profile for a PCR that is perfectly analagous to that of a bacterial culture's growth. In PCR, as in bacterial replication, there is an initial, exponential growth phase followed by a slackening of growth that leads ultimately, upon exhaustion of available resources, to a stationary phase in which there is no additional replication. Quantitation is based on the fact that the more target sequence there is to begin with, the fewer cycles it will take for a PCR to go through these stages. If a threshold level of fluorescence is set in the (detectable) growth phase, one can ask, how many cycles does it take to surpass this threshold? This number of cycles, which can be interpolated to fractions of cycles, is inversely and linearly related to the logarithm of the initial number of target sequences (Higuchi, et al., 1993). 
A concern with the "dye-alone" method of PCR product detection is that non-specific, unintended amplification products will also fluoresce, with the potential of creating falsely positive results. Sometimes, these unintended amplification products arise from partial homologies between the primers and non-target sequences contained elsewhere in the nucleic acid sample. This can usually be overcome by using alternative primer sequences and/or more stringent primer annealing conditions. Many times, however, the unintended amplification product is the result of a primer-extension that uses the other PCR primer as its template (Chou, et al., 1992). This inefficient process creates a dsDNA that has been termed "primer-dimer". It's important to note that primer-dimer, when monitored in realtime, usually arises only after many cycles and does not interfere with the quantification of target sequences unless the target is at relatively low amounts $(<10-1000$ copies $)$. Also, additional means can be taken to significantly delay the appearance of primer-dimer (see section V, "Enhancing specificity and sensitivity", below).

Alternatively, the sensitivity limit imposed by primer-dimer can be overcome by using a specific, fluorescent oligonucleotide probe whose fluorescence increases only upon its binding to a complementary sequence in the target amplification product. (Holland, et al., 1991, Lee, et al., 1993, Heid, et al. 1996, Tyagi, et al., 1997, Wittwer, et al., 1997a). Using probes has the disadvantage of their synthesis and optimization, which can be expensive and time-consuming. In addition, because the number of fluor molecules per molecule of PCR product is one or a few for probe methods versus potentially hundreds for dye-alone methods, the fluorescent signal is inherently weaker, necessitating more sensitive instrumentation. An advantage of the probe methods is the ability to use fluorescent moieties with different emmision spectra for different probes, such that the detection in one amplification of multiple PCR products (different targets or internal amplification controls) is possible by spectral analysis. However, for most researchers requiring simple, specific nucleic acid quantification, a single channel of fluorescence detection and a sensitivity to a few hundred copies per PCR suffices. Additionaly, as described in section VI and VII below, there are both ways to verify that the correct amplification has taken place and have internal amplification controls without using oligonucleotide probes. 
In order to monitor fluorescence emanating from a PCR, a bifurcated fiber-optic cable was first used to both deliver excitation light from a spectrofluorometer to the PCR and to send emmitted light back to the spectrofluorometer (Higuchi, et al., 1992). A fluorescence-monitoring, thermalcycling instrument capable of spectral analysis and based on fiber-optic transmission and a laser light source is now commercially available (Applied Biosystems Model 7700; Perkin-Elmer, Norwalk, CT). In order to monitor 96 PCRs simultaneously, this instrument uses 96 fiber-optic cables and an optical multiplexer to sample rapidly the output of each, one-at-a-time. Mechanically, this becomes increasingly complex and expensive as throughput is further increased. Another approach to monitoring is the use of both photodiode emmitters and detectors. PCRs in small volume (10 microliter), glass capillary tubes are rapidly heated and cooled in an airstream and are interrogated oneat-a-time each cycle during a run (Wittwer, et al. 1997b). A carousel is used to rotate 24 PCRs past the emmitter/detector. Again, obtaining higher throughputs becomes increasingly complex. This

commercially available system (The Light Cycler $^{\mathrm{TM}}$, Idaho Technology, Idaho Falls, ID) is also sensitive enough for the detection of fluorescent probes.

A simpler, less expensive of method of monitoring multiple PCRs is to use a CCD-camera to do quantitative, digital imaging of the PCRs as they sit in the thermocyler block (Higuchi, et al., 1993). With this method, the number of samples monitored is mechanically less relevant; a 384-well sample block can be imaged as easily as a 96-well block. On the other hand - mainly because of the requirement of some distance between the light source and the block and betweens the block and the camera - the sensitivity of camera-based detection is generally less than fiber-optic cable-based detection, which suffers little transmission loss over distance. This lesser sensitivity is generally not a problem for PCR product detection with dye-alone, however.

\section{A CCD-camera based "kinetic thermal cycler"}

The camera system originally described by Higuchi, et al., (1993) has been modified to increase its utility. A compact, light-tight enclosure, fitted over a thermal cycler, avoids the need for a darkroom, requires less space and decreases the distance between the camera and the samples, making the collection of fluorescence more efficient. The use and modification of a commercially available, 
digital imaging system - based on a low-cost, 8-bit video camera and originally intended for recording gel electrophoresis images - significantly reduces the cost and complexity of the system and provides simple-to-use software that automates much of the collection and processing of the data.

A schematic diagram of the system is shown in panel A of figure 1 and a photograph of the system is shown in panel B. The light-tight enclosure, constructed of opaque plastic, is fitted over the sample compartment of the thermal cycler (GeneAmp PCR System 9600, Perkin-Elmer, CT). The heated lid assembly, supplied with the thermal cycler, is not used in this design and is lifted off its track, pulled to the limit of its cable and set on top of the thermal cycler. A mid-range UV lamp, modified to hold two fluorescent tubes, is mounted on top of the optical enclosure to provide $300 \mathrm{~nm}$ excitation of the sample block (model UVM-57, UV Products, San Gabriel, CA.). A solenoid-actuated shutter is mounted under the UV lamp to limit the exposure of the samples to UV light. At the desired times during a PCR cycle, a signal is generated by the controlling software which opens the shutter. The shutter is then held open with reduced current to limit heating of the solenoid. A large, 6"x 5"x 3 $\mathrm{mm}$, dichroic mirror, made of silica, is mounted at $45^{\circ}$ between the UV light and the sample block. It is designed to transmit the $300 \mathrm{~nm}$ excitation light and to reflect the $600 \mathrm{~nm}$ emission, characteristic of ethidum bromide, to the rear of the enclosure (540DCSP, Omega Optical Corp., Brattle Boro, VT). A plastic Fresnel lens with a focal length of 10 inches is mounted between the dichroic mirror and the back of the enclosure and serves to limit the parallax in imaging the samples (part \#32685; 6.7” x 6.7”, Edmond Scientific Co., Barrington, NJ).

The video camera is mounted at the back of the enclosure and pointed at the dichroic mirror. An interference filter with a peak bandpass of $600 \mathrm{~nm}$ is fitted on a $12.5-75 \mathrm{~mm}$, zoom lens. The camera output is sent to a frame-grabber board in a PC computer where the images of the samples are evaluated for sample fluorescence as described below. The video camera, frame-grabber, PC and controlling software are a modification of the Alpha Innotech Digital Imaging System, IS-1000 (San Leandro, CA). While the thermal cycler and controlling PC operate independently, their activities are coordinated by using the thermal cycler's output to a printer - intended to log the completion of cycles - to signal the appropriate time to image the samples. 
Amplification reactions for kinetic analysis are setup with several slight modifications that together compensate as much as possible for the absence of the heated lid. Each reaction is overlaid with $50 \mu 1$ of light mineral oil to prevent evaporation. Small air bubbles trapped under the oil overlay will expand and contract during thermal cycling and can cause signal variations. The set of reactions is therefore centrifuged in a swinging bucket rotor, at about 1,500 RPM, for a minute to insure that all air bubbles are driven out. After the array of samples is set into the sample block of the thermal cycler, a black plastic mask with holes corresponding to the 96-well format is placed over the samples to block a strong signal from any one sample interfering with adjacent samples. A UV transparent plastic cover is placed over the samples which, together with the mask, provides an insulating, convective heat barrier.

\section{Generation of PCR growth curves}

\section{A. Setup for digital imaging}

Once samples are in place and the enclosure closed, the shutter to the UV light can be opened and a sample images taken and displayed on the PC monitor at varying lengths of exposure. The basal fluorescence from etbr in the samples can be seen in the images. Using the imaging software, the individual samples are indicated by overlaying an array of 96 circular "Areas of Interest", or AOIs, over the image (figure 2). The array of AOIs may be moved, and if necessary distorted, to more exactly match the sample positions in the captured image. The circle size can be varied but is kept constant within any one array. Analog to digital conversion of CCD pixel voltages is accomplished in the frame grabber board described above. The eight-bits of digital resolution result in 256 levels of light measurement at each pixel. During a run the software sums all the pixel measurements within each AOI and records the values.

The data stream from a simple kinetic PCR experiments is a series of such sample-specific fluorescence measurements over the course of thermalcycling. Although such measurements can be taken at all stages of a PCR cycle (Wittwer, et al., 1997a), most frequently the measurements focus on the extend phase (or the anneal/extend phase if monitoring a "two-temperature" PCR). It is during these phases that double-stranded DNA is made and indicator dyes can stably bind. Typically for each 
PCR there is a single fluorescence measurement towards the end of this phase for each cycle of PCR. With 96 PCRs the total number of fluorescence measurements $=96$ times the number of PCR cycles (typically 30 to 60). A straightforward way of handling such a data matrix is with a spreadsheet program for personal computers such as Microsoft's Excel, which can tabulate and graph for each PCR a fluorescence growth curve (figure 3).

\section{B. Normalization of fluorescence measurements}

In order to generate growth curves for which meaningful comparisons can be made, two levels of normalization are needed. These are (1) the normalization of differences in fluorescence measurement which occur from cycle-to-cycle, and (2) the normalization of measurement differences which occur from sample-to-sample. Cycle-to-cycle variation is caused by drift and flicker of the UV lamp and by photo-bleaching of the fluorescent dye. Sample-to-sample variation has many sources. In particular, there is non-uniformity of illumination which arises from the dichroic mirror and it's incident-angle-dependent transmission of the UV light. This results in stronger illumination in the center of the sample block. In addition there are differences in the transmissiveness of caps and oil overlays over each sample.

To correct for cycle-to-cycle variation, an invariant fluorescence standard or standards are used in the image field. Deviations in the measured fluorescence of these standards are corrected (to equal the average the of measurement for all cycles) by using cycle-specific normalization factors. These factors are then applied to each cycle's measurements of all samples (figures $3 \mathrm{a}$ and $3 \mathrm{~b}$ ). Such standards work best if their fluorescence is about the same as the initial fluorescence of the monitored PCRs. A simple way to accomplish this is to make a few extra PCRs and inhibit them by leaving out a critical component or by adding EDTA in excess of the divalent cation concentration.

Sample-to-sample variation is corrected for by calculating the relative increases in fluorescence over initial values (figure $3 \mathrm{c}$ ). Using relative rather than absolute measurements accommodates sources of fluorescence variation, such as variable illumination intensity, that result in a constant 
percent attenuation or enhancement of fluorescence. It also sets the baselines of all growth curves to the same average value of approximately one.

Because there can be growth curves with baselines that exhibit slight "drift" upwards or downwards, it's best to use as the "initial" fluorescence level fluorescence measurements from cycles that are are as near as possible to the first cycle at which significant increase is seen. Most of the initial fluorescence level is due to the unbound dye and to dye bound to the ssDNA primers. Until greater than about $50 \mathrm{ng}$ of PCR product/100 $\mu \mathrm{l}$ is generated, there is no significant increase in fluorescence. For most PCRs of experimental value, the generation of $50 \mathrm{ng}$ of PCR product is not expected for at least 12 cycles. On the other hand, the first few cycles are subject to significant fluctuations due to outgassing of bubbles. Therefore an average of each PCR's fluorescence values from cycles 6 to 12 is usually used as the initial fluorescence level.

C. Considerations when applying relative fluorescence measurement

When applied correctly, relative fluorescence measurement results in nearly identical growth curves for replicate PCRs, and in parallel growth curves for PCRs of the same target begun with different target concentrations (figure 3c). The correct application requires that the signal response of the CCD be linear over the range of measurements from beginning to end of amplification. If kept within their dynamic range, CCDs generally have quite linear response. To keep within this dynamic range, the camera gain and exposure time used in a run must not be set so low at the beginning of the run that some pixels do not register, nor so high that at the end of the run some pixels are saturated. The image analysis software allows the false-coloration of pixels registering either lowest (green) or highest (red) levels. Using these false colors, a general calibration of gain and exposure can be done against fluorescence standards of known dye or dye/DNA concentrations. Once camera gain is set, it is usually not changed. When setting up a given experiment, the exposure is set at twice that at which green pixels are seen in any of the wells. This allows enough "headroom" to accomodate fluorescence increases seen using etbr without risk of saturating pixels.

The correct application of relative fluorescence must also take into account non-sample contributions to baseline fluorescence. These include dark current (the accumulation of charge in a 
$\mathrm{CCD}$ in the absence of light), stray light entering the system, and the occasional reaction tube that has a marked endogenous fluorescence (see section VIII for method of prescreening reaction tubes). All of these can can contribute to fluorescence measurement to different extents for different samples. If these contributions are significant compared to the actual fluorescence from the sample, significant error in relative fluorescence measurement can result. In the systems described above, dark current can be easily measured before each run with the excitation lamp off and shutter closed using the same exposure that will be used for the experiment. These dark current values for each well can then be subtracted from subsequent, in-run values. Stray-light should be minimized as much as possible by using non-reflective surfaces and baffling. If still significant, it can be measured for each run and subtracted, just as can be dark current, by putting a non-reflective, black field over the thermal cycler block and taking an image - again at the same exposure used during the run. Note that this measurement will also include dark current contribution.

Incorrect results may also arise when different PCRs in the same run have markedly different compositions. For example, PCRs begun with greater than about $200 \mathrm{ng}$ of genomic DNA have a higher initial fluorescence and a reduced relative fluorescence gain compared to the same amplification of lesser amounts of genomic DNA. This is despite the denaturation of DNA after the initial thermal cycle and is due probably to a proportion of quickly reassociating "snapback" DNA sequences. There can also be differences between PCRs in ionic strength, $\mathrm{pH}$, and co-solvents that result in differential quenching of fluorescence. These differences can result from design or from carryover from sample preparation. There is also the possibility of carryover from sample preparation of fluorescent or fluorescence quenching substances. Sample preparation procedures which ensure high purity of nucleic acids are therefore recommended.

\section{Quantitative analysis}

Once normalized growth curves have been generated, a single threshold level of fluorescence can be set for quantitative comparisons among the PCRs (figure 3c). The threshold is set high enough so that it is not inadverdently crossed by fluctuation or drift in the baseline, but not so high that the linear phase of any growth curve is exceeded. The number of cycles it takes for each growth curve to 
surpass this threshold can be calculated by first fitting a regression line to the fluorescence measurements contiguous to the threshold. We have most frequently used the two contiguous measurements below and the three above for this line. From the equation of this line, a fractional cycle value $\left(\mathrm{C}_{\mathrm{t}}\right)$ is calculated that intercepts the threshold.

$\mathrm{C}_{\mathrm{t}}$ values from PCRs containing target template of known concentration are used to establish a standard quantitation curve. Such a curve is shown in figure 4, based upon the $\mathrm{C}_{\mathrm{t}}$ 's derived from figure $3 \mathrm{c}$, which demonstrates a log-linear relationship between target copy-number and $\mathrm{C}_{\mathrm{t}}$. The occurrance of template-independent PCR product (primer-dimer), is delayed enough that the log-linear relationship persists down to 10 target copies. The equation for such a standard curve can simply be obtained by regression, and the target copy-number of "unknowns" determined by entering into the equation the unknown's $\mathrm{C}_{\mathrm{t}}$. Under optimal conditions, replicate PCRs at greater than a few hundred copies per PCR show a reproducibility in $\mathrm{C}_{\mathrm{t}}$ of about \pm 0.2 cycle, which translates to a copy number reproducibility of about $\pm 15 \%$. Replicate PCRs with less than about 100 copies begin to show additional variation in $\mathrm{C}_{\mathrm{t}}$ due to stochastic fluctuation.

The slope of the log-linear range of the standard curve also reflects the initial DNA replication efficiency of the PCR. Given two otherwise identical PCRs starting at different target concentrations, the slope derives from the number of cycles it takes for the PCR with less target to catch up, in product copy-number, to the other PCR. For example, if there is $100 \%$ replication efficiency (a doubling in copy-number per cycle), it takes exactly one cycle for a PCR begun with a two-fold dilution of starting target to catch up. Lower efficiencies result in larger cycle offsets. This offset, once established, persists over the whole growth curve even as the per-cycle replication efficiency diminishes as the PCRs progress through different phases, from exponential to linear (where the threshold is) to plateau. Note that there is a presumption that the threshold level represents for each growth curve the same amount of product. This will be true if relative fluorescence measurement is applied correctly as described above.

This efficiency can be calculated from the slope of the standard curve (figure 4). In the first cycle of PCR, the resultant copy number of product, $\mathrm{c}_{1}$, is related to the initial target copy number, $\mathrm{c}_{0}$, by: 


$$
\mathrm{c}_{1}=\mathrm{c}_{0}(1+\text { efficiency })
$$

By converting to logorithms and rearranging, it can be seen that:

$$
\begin{gathered}
\log \mathrm{c}_{1}=\log \mathrm{c}_{0}+\log (1+\text { efficiency }) \\
\log \mathrm{c}_{1}-\log \mathrm{c}_{0}=\log (1+\text { effciency })
\end{gathered}
$$

Since $\log c_{1}-\log c_{0}$ is equivalent to the negative slope of the standard curve, this can be restated as:

$$
\begin{gathered}
\text {-slope }=\log (1+\text { efficiency }), \text { or } \\
10^{\text {-slope }}-1=\text { efficiency }
\end{gathered}
$$

When quantitating a large number of different targets at once, it is inconvenient to run standard curves for each target. Kang, et al. (1998) have shown that a single quantitative standard can suffice for the RT-PCR-based quantitation of large numbers of different RNA transcripts. Compared to an outside reference, the measured concentrations were comparable to within two-fold. This could only be true if there were no large discrepencies between the RT and DNA amplification efficiencies of different transcripts. Keeping the amplicon size to less than $300 \mathrm{bp}$ and screening primer sets for efficient DNA amplification, determined as described above, helped accomplish this.

\section{Enhancing specificity and sensitivity}

As discussed above, the primary limit to the sensitivity of dye-alone kinetic PCR is the generation of a template-independent, dsDNA artifact commonly referred to as primer-dimer (Chou, et al., 1992). This artifact is the result of one primer serving as the template for chain-extension of the other, even though there is no site to which the other primer can anneal. This is a relatively rare event; but once such an extended primer is generated, it contains both primer-annealing sequences and thus can serve as a legitimate template for PCR amplification. The generation of such an extended primer is more likely the lower the temperature, perhaps as a result of metastable basepairing of the 3' end of one primer to the other primer.

A significant reduction in primer-dimer was seen if an essential reagent, such as the polymerase, was left out when setting up the PCRs and was only added when the temperature was as high as possible just before the first denaturation step of thermocycling (Mullis, 1991). Such a "hot- 
start" has been made most convenient through the use of a chemically-modified DNA polymerase (AmpliTaq Gold ${ }^{\mathrm{TM}}$, Perkin-Elmer, Norwalk, CT; Birch, 1996) that requires an incubation at greater than 90 deg C. to be activated. This allows the enzyme to be present during PCR setup. For DNA amplifications, the use of the thermally-activated polymerase commonly delays the $\mathrm{C}_{\mathrm{t}}$. for primerdimer to greater than 45 cycles - more cycles than an amplification from a single DNA template copy would require.

An additional hot-start comes about as an ancillary effect of the use of the enzyme uracil-nglycosylase (UNG) and the incorporation dUTP in place of dTTP that is intended to help control carryover-contamination by PCR product (Longo, et al., 1990; Persing and Cimino, 1993). Illegitimate primer extensions that incorporate $\mathrm{dU}$ are cleaved by $\mathrm{UNG}$ until the temperature is raised to greater than 50 deg. C., which inactivates the UNG. For DNA amplfications, we have found that the thermally-activated polymerase and UNG hot-starts, when combined, can delay primer-dimer further than either hot-start alone. The UNG hot-start alone is of significant benefit for single-tube, singleenzyme (e.g., Tth polymerase; Myers, et al., 1995) RNA amplifications, for which a thermallyactivated polymerase is unavailable.

For DNA and RT-PCR we have found that both primer sequence and primer quality can affect the occurance of primer-dimer. R. Saiki (personal communication) has shown that primer-dimer is usually reduced when both primers have $3^{\prime}$ ends that terminate in a series of at least two dA residues. For this reason, it is worthwhile to choose amplification primers which have such termini, and at the same time, yield as small an amplicon as possible to maintain as high an RT and DNA amplfication efficiency as possible. Additionally, different synthesis's of the same primer sequences can yield markedly different levels of primer-dimer in PCR. For this reason, the resynthesis of primers that give high levels of primer-dimer can sometimes help. The growth curves shown in figure 3 , in which the $\mathrm{C}_{\mathrm{t}}$. for primer-dimer is greater than 45 cycles, were generated by using these suggestions for primer design in single-tube RT-PCRs using Tth polymerase and dU incorporation with UNG.

\section{Verification of PCR product identity without probes}


Using dye detection, amplification of the desired target sequence can be validated in two ways. First, under the right conditions, the level of fluorescence reached at the plateau phase of a PCR growth curve is proportional to the amplicon size. Thus, amplification of anything other than amplicon of the expected size for the specific target results in an unexpectedly high or low plateau fluorescence. Second, it is possible to perform DNA melting analysis in a thermocycling instrument based upon the ability of these dyes to distinguish between ss and dsDNA (Ririe, et al., 1997). This allows one to distinguish specific from non-specific amplfication products based upon the melting temperature of the duplexes.

The relationship between fluorescence and amplicon size was first noted in "primer-limited" PCRs. The amount of PCR product that can be made is limited by the starting primer concentration when it is less than about $0.4 \mu \mathrm{M}$ each (Higuchi, et al., 1993). One result of such primer limitation is a growth curve with a very flat plateau phase like those in the growth curves of Figure 3 . The lack of additional growth probably results when the concentration of product exceeds that of remaining primer by enough such that product reassociation occurs before primer annealing and extension can. Since the concentration of product at which this occurs is mostly independent of the size of the amplicon, amplicons of different molecular weight "plateau" at the same concentration. This means that the mass of dsDNA made at plateau is proportional only to the amplicon size. With etbr at $4 \mu \mathrm{g} / \mathrm{ml}$, the fluorescence of dsDNA is proportional to amplicon mass up to at least $4 \mu \mathrm{g}$ dsDNA/100 $\mu 1$ PCR (Higuchi, et al., 1993). Taken together, these proportionalities predict that the plateau fluorescence should be a direct measure of amplicon size.

This can be seen in the growth curves shown in figure $3 \mathrm{c}$ in which the product present in the no template reactions - low molecular weight primer-dimer - plateaus at a much lower relative fluorescence that does the specific target product that is more than twice as large. Mixtures of primerdimer and target product show intermediate fluorescence levels. Detecting primer-dimer in this way has so far been the most useful role for this mode of analysis.

More generally, Fig 5 shows the directly proportional relationship between amplicon size and relative fluorescence at plateau for six different amplicons ranging in size from about $100 \mathrm{bp}$ to $550 \mathrm{bp}$. 
When relative fluorescence measurement is correctly done as described above, it is possible to distinguish amplicons that differ by more than about $20 \%$ in size.

Ririe, et al. (1997) have shown in their kinetic PCR instrument that by slowly denaturing or annealing PCR product after kinetic PCR with SYBR green detection, they can follow the melting or reannealing of the duplex DNA. The temperature at which this occurs is a function of the sequence of the duplex. Primer-dimer, because of its short length, generally has a melting temperature $\left(\mathrm{T}_{\mathrm{m}}\right)$ well below that of the correct target sequence. It is usually possible to resolve small amounts of target amplicon in the prescence of large amounts of primer-dimer. The closer the $\mathrm{T}_{\mathrm{m}}$ ' $\mathrm{s}$, the more difficult is the resolution; it is possible to resolve equal amounts of amplicon that differ by about $2 \mathrm{deg} .{ }^{\circ} \mathrm{C}$ in $\mathrm{T}_{\mathrm{m}}$. Such melting temperature analysis has been performed using etbr on the CCD-based instrument.

\section{Detecting inhibition}

Inhibition of the PCR can cause falsely negative results or underestimation of the target sequence concentration. However, the shape of kinetic PCR growth curves can indicate whether or not this is occuring. Partial inhibition usually results in a growth curve that is shallower than expected (Higuchi, et al., 1993) resulting in a delayed Ct. Total inhibition results in a flat growth curve profile, which is an obvious indicator of inhibition when at least primer-dimer is expected; but if no primerdimer is expected this does not distinguish a totally inhibited PCR from a truly negative one. Another "gray area" can be a partially inhibited PCR displaying a growth curve that is delayed to where primerdimer would be expected. Usually, however, the exact shape of the inhibited growth curve is still distinctive.

Since these methods of detecting inhibition are subjective and qualitative, it would be preferable to develop a method that is analogous to the use of an internal quantitative standard, or "mimic" (Ferre, F., 1992) that would allow the calculation of the correct target copy number even with the occurance of partial inhibition. An approach to this is shown in Figure 6. An internal standard template in known, high amount is added to the PCR. Primers to this template are used at a low, limiting concentration. If target sequence is present, the amplification results in a biphasic growth curve as shown, the first phase being the primer-limited amplification of the control template, the 
second being target amplfication. Presence of an inhibitor would delay or eliminate the first phase. The target phase would also be delayed in a way that could be related to the delay of the control phase. Knowing this relationship, a correct quantitation could still be performed. Figure 6 shows the effect of $3 \%$ and $5 \%$ methanol. A total inhibition with 5\% methanol eliminates both phases of the growth curve; a partial inhibition with $3 \%$ delays the two phases.

It has been problematic so far to obtain an internal control template of this type whose response to inhibitor can mimic that of the target well enough for accurate quantification. It may be that the best use of this type of control will be to design it such that it is always more sensitive to inhibition than is the target sequence. This would provide a "red-flag" indicating that the inhibitors are present and that repurification of the sample is necessary to obtain valid quantification.

\section{Protocol}

This protocol is for a RT-PCR using rTth enzyme (Myers, et al., 1995), UNG with dUTP incorporation, and a $50 \mu 1$ sample addition.

Reagents

$\begin{array}{ll}5 X \text { buffer } & 0.25 \mathrm{M} \text { Tricine-HCl, } \mathrm{pH} 8.3, \\ & 0.6 \mathrm{M} \text { potassium acetate, } 25 \% \text { glycerol } \\ \text { dNTP mix } & 20 \mathrm{mM} \text { each dG-, dA-, and dCTP, } \\ & 2 \mathrm{mM} \text { dTTP and } 20 \mathrm{mM} \text { dUTP } \\ \text { UNG } & 1 \mathrm{U} / \mu \mathrm{l}(\text { Perkin-Elmer, Norwalk, CT) } \\ \text { etbr } & 100 \mu \mathrm{g} / \mathrm{ml} \text { in } \mathrm{H}_{2} \mathrm{O} \\ \text { Tth DNA polymerase } & 2.5 \mathrm{U} / \mu \mathrm{l}(\text { Perkin-Elmer, Norwalk, CT) }\end{array}$

1. Prescreen all the MicroAmp ${ }^{\mathrm{TM}}$ tubes (Perkin-Elmer, Norwalk, CT) and caps, if used, by spreading the tubes out on a UV transilluminator covered with plastic wrap. Remove any tubes that show strong fluorescence.

2. Program the thermal cycler as follows and save for further use as a series of linked files 


$\begin{array}{lll}50{ }^{\circ} \mathrm{C} & 2 \mathrm{~min} & \text { UNG incubation step } \\ 60{ }^{\circ} \mathrm{C} & 30 \mathrm{~min} & \text { Reverse transcription step } \\ 95^{\circ} \mathrm{C} \quad 30 \mathrm{sec} & \text { Denaturation step } \\ 55^{\circ} \mathrm{C} \quad 30 \mathrm{sec} & \text { Anneal step } \\ 72^{\circ} \mathrm{C} \quad 30 \mathrm{sec} & \text { Extend step }\end{array}$

(Set controller to take image 25 seconds into this extend step)

Repeat last three steps for desired number of PCR cycles.

$4{ }^{\circ} \mathrm{C}$ Hold or $72{ }^{\circ} \mathrm{C}$ Hold

3. Assemble the TC9600 tray and rack. Place the required number of screened PCR tubes in the rack including tubes for positive and negative controls and for fluorescence standards. The fluorescence standard is made by adding 50ul of a 40mM EDTA solution to 50ul of the Master Mix (step 4).

4. Prepare the Master Mix as follows - listed is the volume of each component to add to the Master Mix per single 100 ul reaction:.

$\begin{array}{ll}\text { distilled } \mathrm{H} 2 \mathrm{O} & 14.5 \mu \mathrm{l} \\ \text { 5X Buffer } & 20 \mu \mathrm{l} \\ \text { dNTP mix } & 2.5 \mu \mathrm{l} \\ \text { UNG } & 2 \mu \mathrm{l} \\ 25 \mu \mathrm{M} \text { forward primer } & 1 \mu \mathrm{l} \\ 25 \mu \mathrm{M} \text { reverse primer } & 1 \mu 1 \\ \text { etbr } & 1 \mu 1 \\ \text { Tth DNA polymerase } & 4 \mu \mathrm{l} \\ 0.1 \mathrm{M} \mathrm{Mn}(\mathrm{OAc})_{2} & 4 \mu 1\end{array}$

The Master Mix can be prepared in advance by adding all the components except the $\mathrm{Mn}(\mathrm{OAc})_{2}$.

5. Thoroughly mix the Master Mix immediately after adding the $\mathrm{Mn}(\mathrm{OAc})_{2}$, and pipette $50 \mathrm{ul}$ of the Master Mix into each reaction tube. Add 50 microliters of sample to each PCR tube. Avoid introducing air bubbles.

6. There are two options for preventing refluxing of the samples. They can be covered with one drop of mineral oil, then the rack of samples spun in a centrifuge in a swinging bucket rotor (1500 rpm 
for $1 \mathrm{~min}$.$) to remove tiny air bubbles that otherwise congeal into large air bubbles and intefere with$ detection as the samples heat. Or, add one AmpliWax ${ }^{\mathrm{TM}}$ Gem 100 (Perkin-Elmer, Norwalk, CT) to each tube, being careful to add only one bead. Tube caps may be used, but we find it convenient not to use them at all, being careful to avoid jostling of the rack. Once melted and hardened, the Ampliwax barrier itself seals the reaction tubes.

7. Transfer the rack to the kinetic thermal cycler and insure that the tubes are seated in the block. Set the exposure (see section IVc, above) and position the grid over the sample image and start the analyzing software. Make sure that the kinetic PCR software is "looking" for the right Setpoint\# in the run set up dialog box. For a three temperature thermal cycle, the software should be set for "Setpt\#3". Start the programmed series of thermal cycles on the thermal cycler.

\section{Acknowledgements}

We wish to acknowledge the contributions of Carita Elfstrom, Mary Fisher and Stanley Wang who performed much of the experimental work, and of Rob Watson and Tom Vess who helped with data analysis. We also wish to thank Darryl Ray and Haseeb Chaudry for their help with the imaging system. We thank John Sninsky for his long-standing support of this technology.

\section{References}

Birch, D.E., (1996). Simplified hot start PCR. Nature; 381, 445-446.

Chou, Q., Russell, M., Birch, D.E., Raymond, J., and Bloch, W. (1992). Prevention of PCR mispriming and primer dimerization improves low copy-number amplifications. Nucl. Acids Res. 20, $1717-1723$.

Ferre, F. (1992) Quantitative or semi-quantitative PCR: reality versus myth. PCR Methods Appl. 2, 1-9. Heid, C.A., Stevens, J., Livak, K.J., and Williams, P.M. (1996) Real time quantitative PCR. Genome Res. 6, 986-994

Higuchi, R., Dollinger, G., Walsh, P.S., and Griffith, R. (1992). Simultaneous amplification and detection of specific DNA sequences. Bio/Technology 10, 413-417. 
Higuchi, R., Fockler, C., Dollinger, G, and Watson, R. (1993). Kinetic PCR analysis: real-time monitoring of DNA amplification reactions. Bio/Technology 11, 1026-1030.

Holland, P.M., Abramson, R.D., Watson, R., and Gelfand, D.H. (1991). Detection of specific polymerase chain reaction product by utilizing the $5^{\prime}$ to 3 ' exonuclease activity of Thermus aquaticus DNA polymerase. Proc. Natl. Acad. Sci. 88, 7276-7280.

Kang, J.J., Watson, R.M., Fisher, M.E., Higuchi, R., Gelfand, D.H., and Holland, M.J. (1998) Transcript quantitation in total yeast cellular RNA using kinetic PCR . submitted.

Lee, L.G., Connell, C.R., and Bloch, W. (1993) Allelic discrimination by nick-translation PCR with fluorogenic probes. Nucleic Acids Res. 21, 3761-3766

Longo, M.C., Berninger, M.S., and Hartley, J.L. (1990). Use of uracil DNA glycosylase to control carry-over contamination in polymerase chain reactions. Gene 93,125-128.

Mullis, K.B. (1991). The polymerase chain reaction in an anemic mode: how to avoid cold oligodeoxyribonuclear fusion. PCR Methods Appl. 1, 1-4.

Myers, T.W., and Sigua, C.L. (1995). Amplification of RNA: high-temperature reverse transcription and DNA amplification with Thermus thermophilus DNA polymerase. In "PCR Strategies" (J. Sninsky, et al., Ed.), pp.58-68. Academic Press, San Diego.

Persing, D.H., and Cimino, G.D. (1993) Amplification product inactivation methods. In "Diagnostic Molecular Microbiology: Principles and Applications” (D.H. Persing, et al., Ed.), pp. 105-121. American Society for Microbiology, Washington, D.C.

Ririe, K.M., Rasmussen, R.P., Wittwer, C.T. (1997) Product differentiation by analysis of DNA melting curves during the polymerase chain reaction. Anal Biochem. 245, 154-160.

Tyagi, S., Bratu, D.P., and Kramer, F.R . (1998) Multicolor molecular beacons for allele discrimination. Nat. Biotechnol. 16, 49-53.

Wittwer, C.T., Herrmann, M.G., Moss, A.A., Rasmussen, R.P. (1997a) Continuous fluorescence monitoring of rapid cycle DNAamplification. Biotechniques 22, 130-131

Wittwer, C.T., Ririe, K.M., Andrew, R.V., David, D.A., Gundry, R.A., and Balis, U.J. (1997b) The LightCycler: a microvolume multisample fluorimeter with rapid temperature control. Biotechniques 22, 176-181 


\section{Figure 1}

A) Schematic diagram of light-tight enclosure that fits over the sample block of a thermal cycler. The light path is indicated from the UV lamp, through the dichroic mirror to illuminate the sample block; and then from the sample block, reflected from the dichroic mirror, through the Fresnel lens and interference filter to the CCD camera. B) The light-tight enclosure mounted on a Perkin-Elmer thermal cycler. The heated lid assembly has been moved aside to accomodate the enclosure.

A

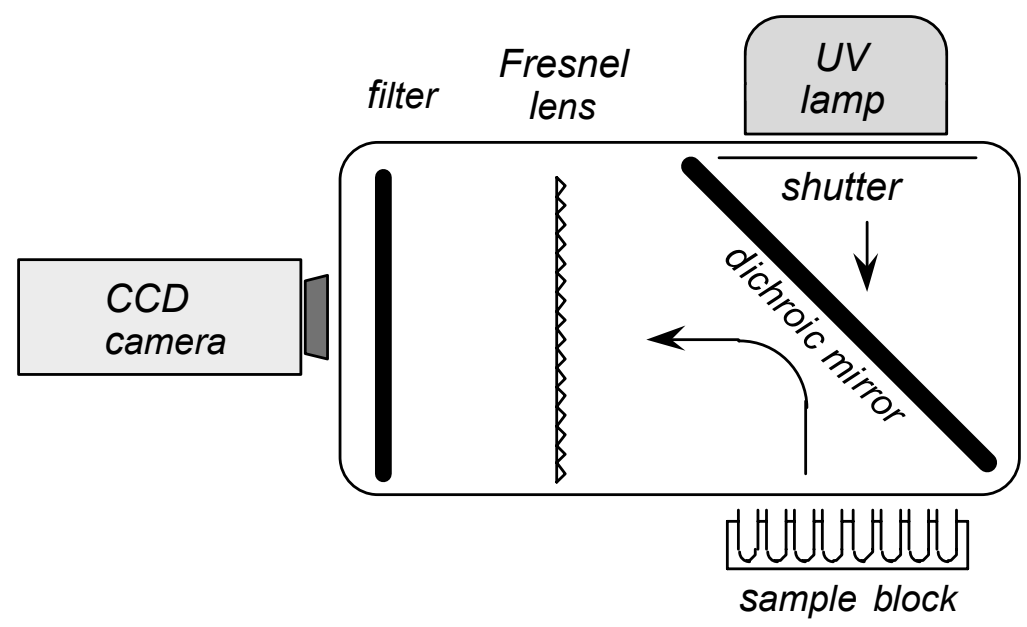

B

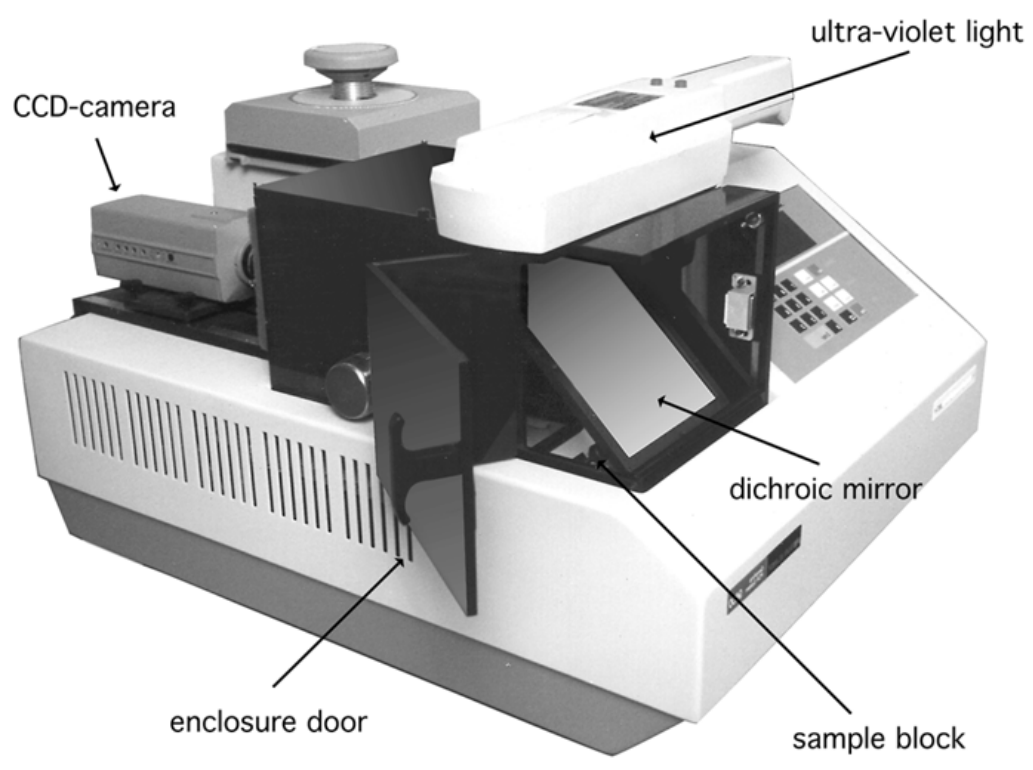




\section{Figure 2}

The user interface for the collection and analysis of video images of an array of samples. A grid of 96 Areas of Interest (AOIs) is shown on the screen as are many user selectable menus and control buttons.

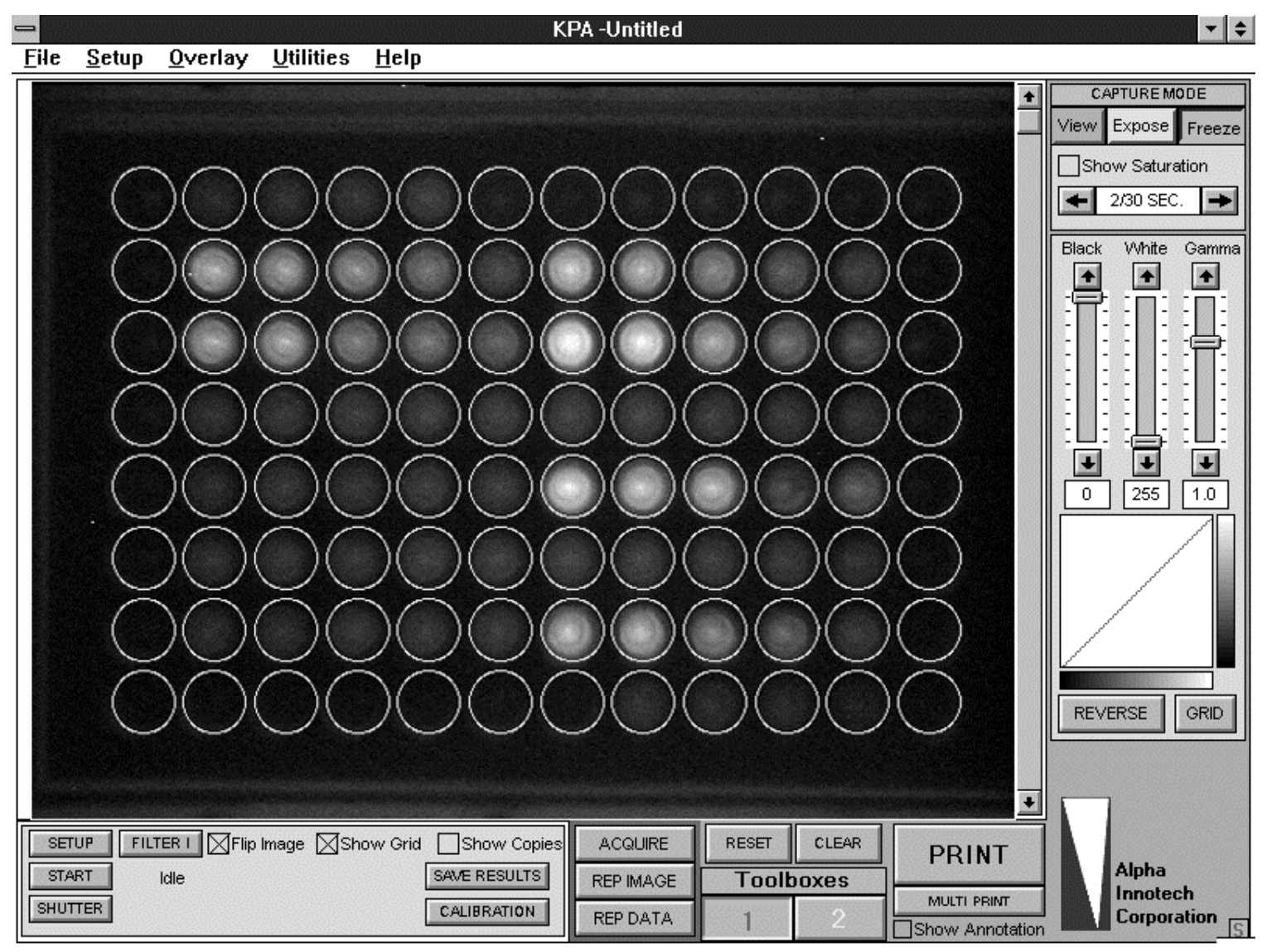




\section{Figure 3}

A. The raw fluorescence output from the CCD-camera based instrument showing growth curves from eight RT-PCRs from the indicated starting copy numbers of HIV RNA template

B. The data from A), cycle-to-cycle normalized as described in the text

C. The data from B), sample-to-sample normalized as described in the text. The threhold level of fluorescence from which $\mathrm{C}_{\mathrm{t}}$ 's (see text) are derived is indicated.

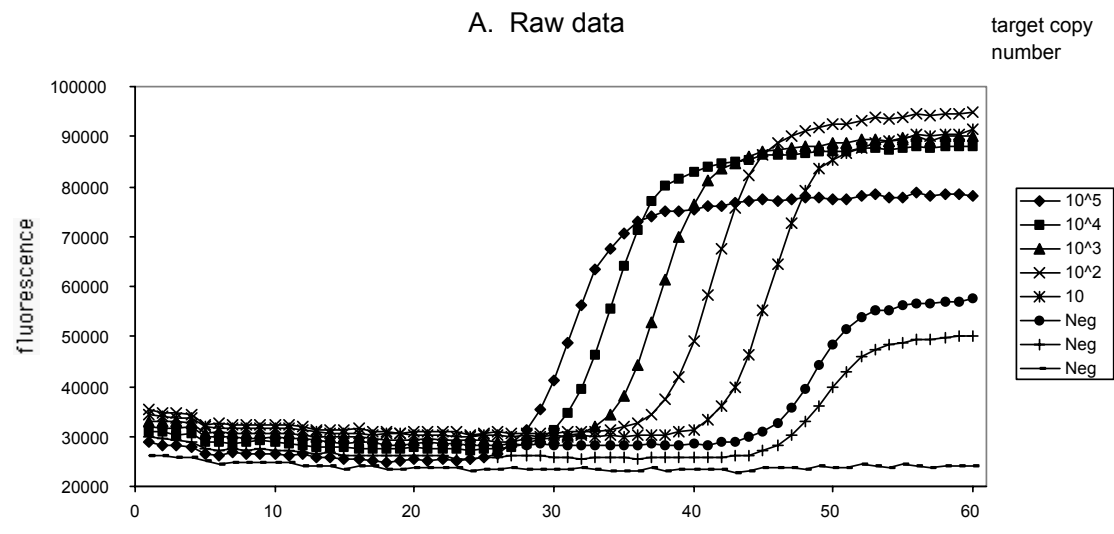

B. Cycle-to-cycle normalized
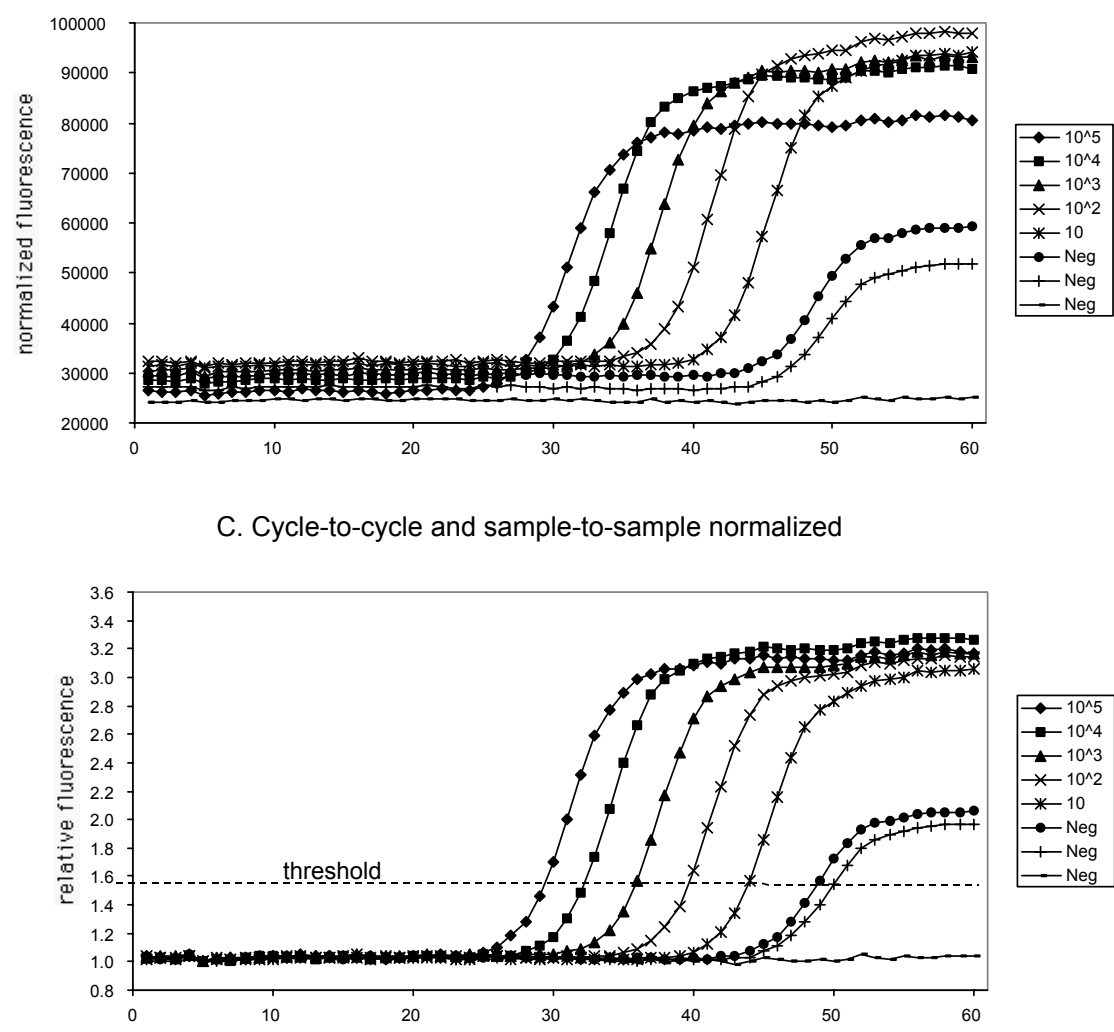


\section{Figure 4}

A standard quantitative curve regressed from the $C_{t}$ 's of the growth curves shown in Figure $3 \mathrm{c}$. A calculation of the initial amplification efficiency from the slope of the standard curve is shown (see text for explanation).

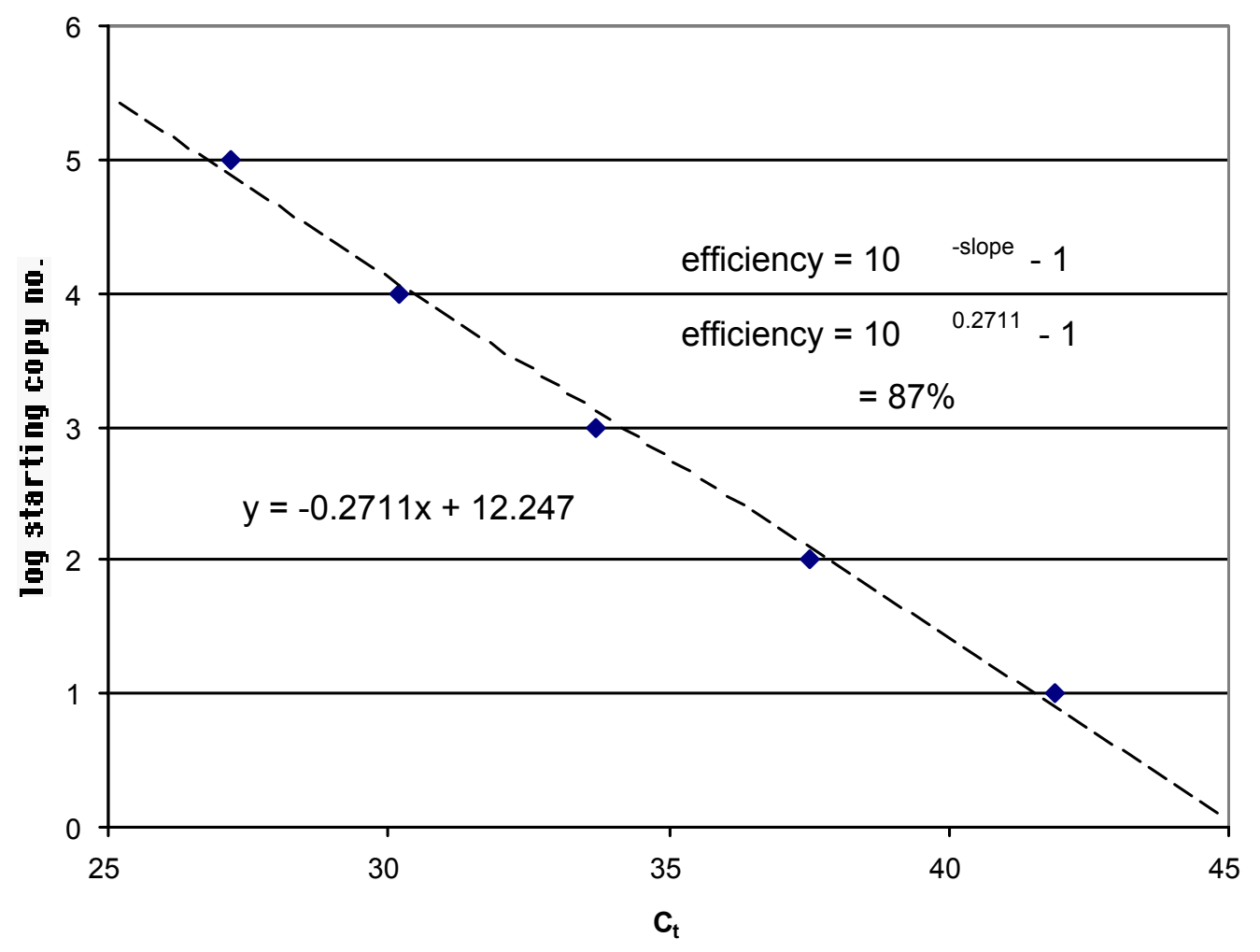




\section{Figure 5}

The relationship between amplicon size and relative fluorescence yield is demonstrated for six different amplicons. In separate PCRs, three forward primers increasingly distant from one common back primer were used on two M13 ssDNA template (with and without a 115 base cloned insert) to generate the six different sized products. The common back primer was always at limiting concentration.

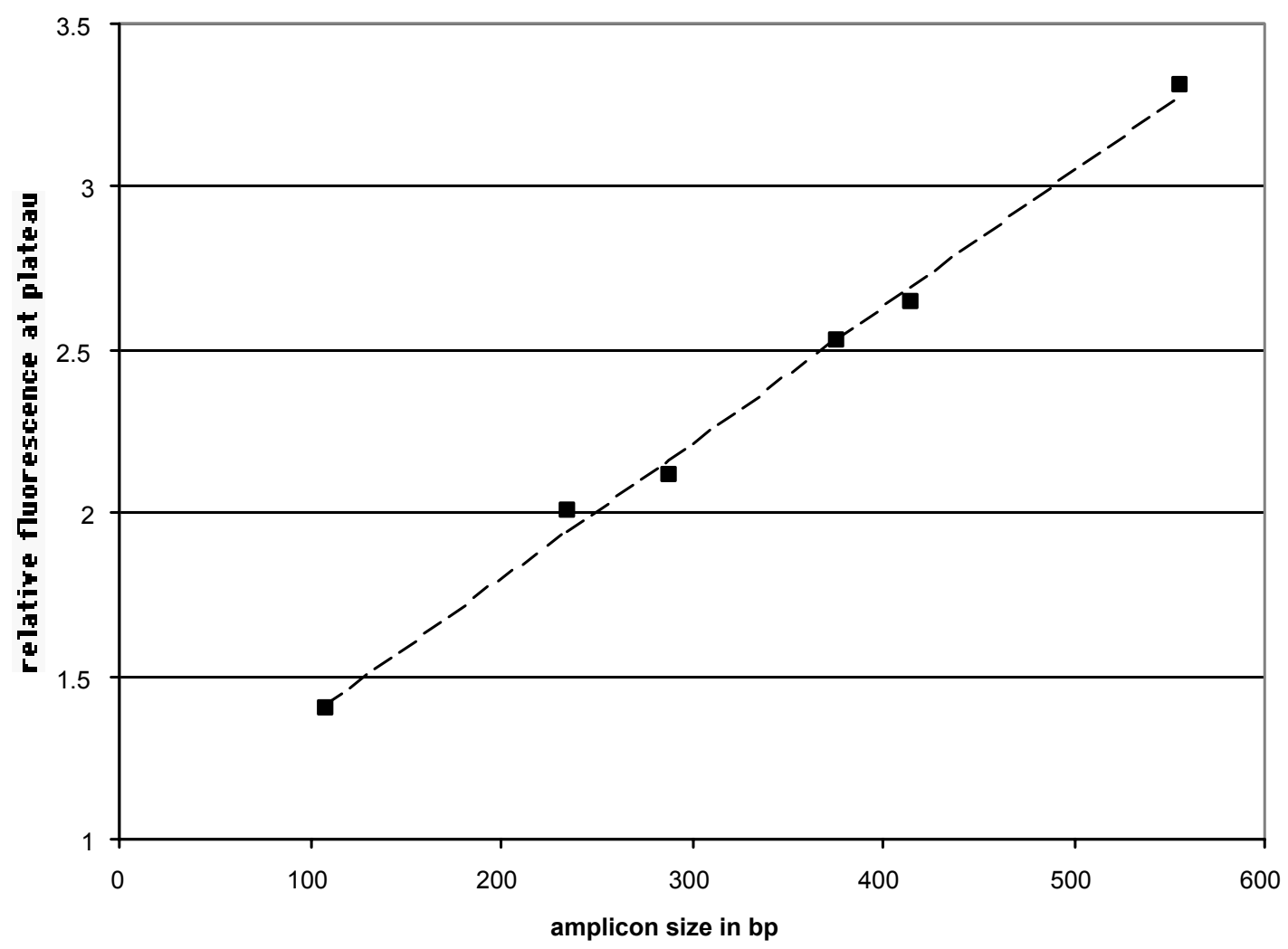




\section{Figure 6}

A high-copy number, internal amplfication control. The biphasic growth curve results from the inclusion, at limiting concentration, of a high-copy number template (M13 ssDNA) and two additional (M13) primers into a PCR directed to an unrelated (human beta-globin), low-copy template. Adding methanol $(\mathrm{MeOH})$ inhibits both phases of the growth curve.

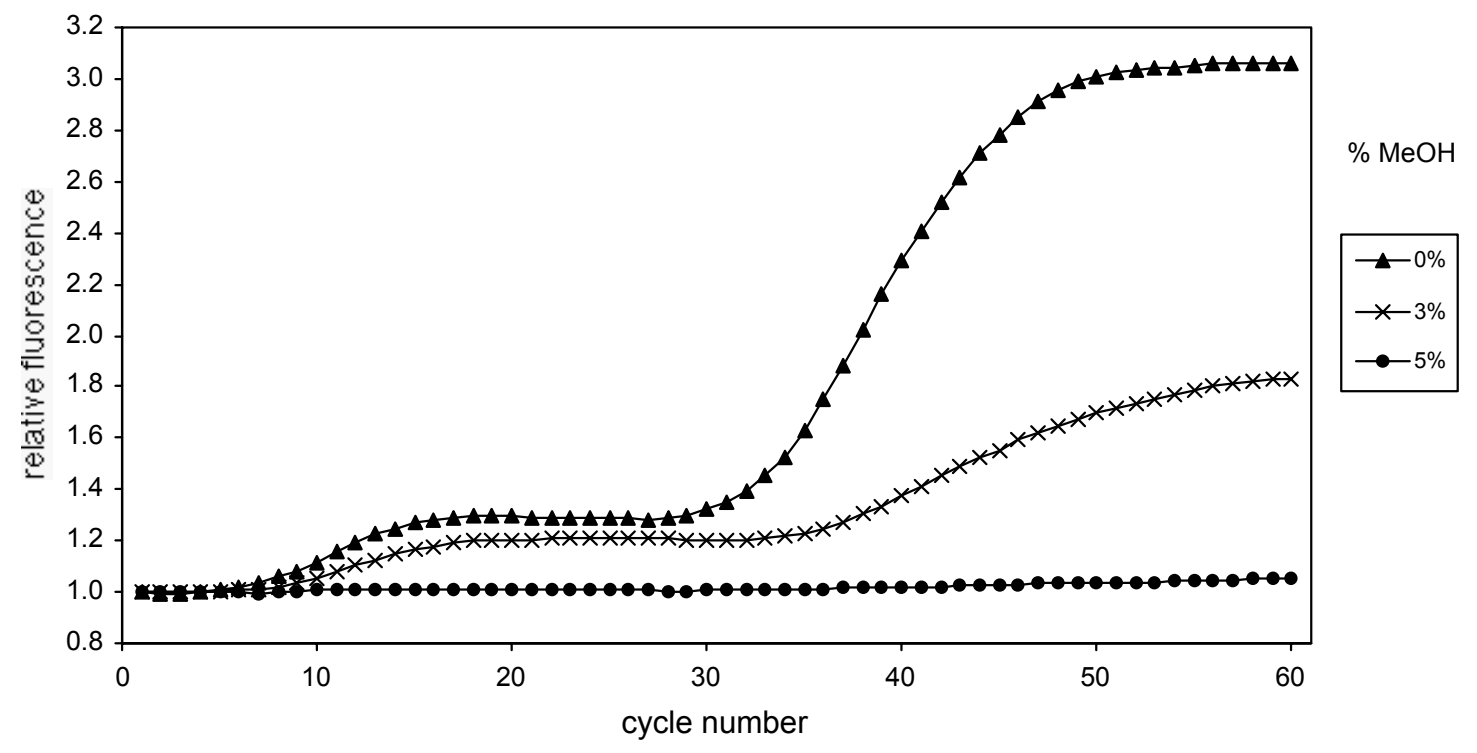

